\title{
Desmopressin, Imipramine, and Oxybutynin in the Treatment of Primary Nocturnal Enuresis: A Randomized Clinical Trial
}

\author{
Maryam Seyfhashemi ${ }^{1, *} ;$ Raheb Ghorbani $^{2}$; Abbas Zolfaghari ${ }^{1}$ \\ ${ }^{1}$ Department of Pediatrics, Semnan University of Medical Sciences, Semnan, IR Iran \\ 2 Research Centre for Social Determinants of Health, Department of Community Medicine, Semnan University of Medical Sciences, Semnan, IR Iran \\ ${ }^{*}$ Corresponding Author: Maryam Seyfhashemi, Department of Pediatrics, Semnan University of Medical Sciences, Semnan, IR Iran. Tel: +98-9121314203, Fax: +98-2333460099, \\ E-mail: maryam_seif@hotmail.com
}

Received: November 14, 2013; Revised: December 4, 2014; Accepted: April 14, 2015

\begin{abstract}
Background: Nocturnal enuresis is the most common pediatric urologic problem in outpatient clinics.
Objectives: To assess the effect of various monotherapies, and comparing the effects of desmopressin, imipramine, and oxybutynin in children with enuresis, as well as the influence of socioeconomic and cultural factors of their families on the response and relapse rates. Patients and Methods: The study was a randomized clinical trial conducted on 92 children aged $5-14$ years, referred to the pediatric clinic of Semnan University Hospital in Semnan, Iran. Children with primary nocturnal enuresis were randomly allocated to three different treatment groups: desmopressin ( $n=30)$, imipramine $(n=31)$, and oxybutynin $(n=31)$ all for 6 weeks. The socioeconomic and demographic characteristics of all participants were recorded. The number of wet nights per week was noted at the end of the 6-week-trial, and children were followed up to three months for relapse.

Results: Children in the oxybutynin group showed a slightly higher response rate (71.0\% success) and a lower relapse rate (31.8\%), while in the desmopressin group the response and relapse rates were $63.3 \%$ and $57.9 \%$, respectively, and in the imipramine group $61.3 \%$ and $63.2 \%$, respectively. However, the difference between the 3 groups in terms of response $(\mathrm{P}=0.701)$ and relapse rates $(\mathrm{P}=0.095)$ was not statistically significant.

Conclusions: There is no significant difference between monotherapy with desmopressin, imipramine or oxybutynin in children with enuresis. However, oxybutynin showed a higher response rate and a lower relapse rate compared to other medications. More clinical trials with a larger sample size are needed to clarify these uncertainties.
\end{abstract}

Keywords: Nocturnal Enuresis; Desmopressin; Oxybutynin; Imipramine

\section{Background}

Nocturnal enuresis or bed wetting is defined as the persistence of spontaneous and inappropriate voiding of urine during sleep after the age of expected bladder control $(1,2)$. Enuresis is explained in the DSM-IV as the "repeated voiding of urine into bed or clothes at least twice a week for at least 3 consecutive months in a child who is at least 5 years of age" (3). Nocturnal enuresis is described as the primary enuresis if the child has not been dry for more than 6 months (4). Bed wetting is the most common pediatric urologic problem in outpatient clinics (1) and is 3 times more common in boys than girls (2). Nocturnal enuresis affects 15 - 20\% of 5 year olds, $5 \%$ of 10 year olds, $7 \%$ of 7 year olds, 2 - 3\% of 12 - 14 year olds, and 1 - $2 \%$ of individuals aged 15 years and over (4). Without treatment, the average annual spontaneous cure rate is approximately $15 \%$ (5).

Enuresis seems to be a multifactorial condition with different contributing factors, including genetics, sleeparousal dysfunction, overnight polyuria, urodynamic features, developmental delay, and psychosocial problems (1). Before considering any therapeutic approach, it is important to identify the possible underlying mechanism of bed wetting and rule out other organic causes of enuresis. Careful evaluation is required to determine specific urologic, psychosocial, developmental, and sleep-related etiologies. However, most children with primary nocturnal enuresis only need a precise medical history, physical examination, and urinalysis before starting the treatment. Management of primary nocturnal enuresis should begin with educating and reassuring the child and parents about the condition. Behavioral interventions are the first line of treatment and pharmacotherapy is only indicated for children who are impacted by their enuresis (6). Medication should not be started in children unless non-pharmacological interventions fail (2). Three frequently used drugs for the treatment of enuresis are imipramine, desmopressin, and oxybutynin $(1,2,7)$.

\section{Objectives}

The study aimed to assess which one of the above pharmacological interventions is more efficacious. We evalu- 
ated the outcomes of interventions to determine: 1) the rate of initial success as defined by the reduction in the number of wet nights per week, and 2) the rate of relapse after discontinuing the medications.

The study, also explored the effect of demographic and socioeconomic variables on the response and relapse rate.

\section{Patients and Methods}

The study was a randomized clinical trial conducted in 92 otherwise healthy children aged 5 - 14 years, who were referred to the pediatric clinic of Amir al Momenin Hospital in Semnan, Iran, during 2009 and 2010. The hospital is a provincial referral general hospital affiliated to Semnan University of Medical Sciences. Inclusion criteria included primary enuresis (two or more wet nights per week and not dry for more than 6 months after the third year of life), not receiving any medication for enuresis during the last 6 months, and lack of clinical or laboratory signs suggestive of any underlying disease other than enuresis. Both children and their parents were given detailed explanation about the study and treatment rationale, and the side effects of the drugs. Then, the parents would be asked to sign the consent form, if they allowed their children to participate in the trial.

A simple random sampling method was used to recruit the patients from the children with enuresis referred to the pediatric clinic of Amir al Momenin Hospital. In this regard, 150 children (5-14 years old) with primary enuresis were recruited. Children were examined by pediatricians to ensure they were otherwise healthy. After detailed history taking and careful physical examination, urinalysis, urine culture, and urinary system, ultrasound were performed for all children. The parents of 19 children refused to participate in the study and other 15 patients withdrew from the study during the first assessment. A further 24 children were excluded from the study because of either history of day wetting, abnormal urinalysis or ultrasound, or urinary tract infection. Therefore, our sample included 92 patients aged between $5-12$ years.

A simple randomization procedure was used to allocate the remaining 92 patients into 3 treatment groups. The first group was given 1-2 puffs (10 - $20 \mu \mathrm{g}$ ) of nasal desmopressin $(n=30)$, the second group 25 - 75 mg imipramine $(\mathrm{n}=31)$, and the third group 5 - $15 \mathrm{mg}$ oxybutynin $(\mathrm{n}=$ 31). Medications were dispensed at the clinic and participants were asked to fill the follow-up forms every morning and attend a follow up visit every 3 weeks. During the first visit, the detailed explanation of the medication, its' possible side effects, dosage, and other necessary information were given, and participants were asked to call the physician if any major side effect was noticed. During the next follow up visits, the children were evaluated for the side effects or any possible difficulties. If participants were affected by the side effects of the medication, the responsible drug would be discontinued. When a child did not show up for the follow up visit, the researchers contacted him or her to find out the reasons and discuss the problems. Any child who had not received the medication properly or was affected by major side effects was excluded from the study. The duration of treatment was 6 weeks and all children were evaluated regarding the number of wet nights per week at the end of the 6th week. Moreover, all children were followed up for a further 3 months for the number of wet nights after discontinuing the medication (Figure 1).

The criterion for successful treatment was lack of wet nights for at least two consecutive weeks. Relapse was defined as the reappearance of more than 2 wet nights per week, 3 months after the discontinuation of pharmacological therapy.

The study protocol was approved by the Ethics Committee of Semnan University of Medical Sciences. The trial has been registered in the Iranian registry of clinical trials (IRCT138807042503N1).

Statistical analysis were performed by KolmogorovSmirnov, Mann-Whitney, and Chi-square tests to compare demographic and socioeconomic variables, cure rate and relapse rate between the 3 groups using SPSS 16.0 software. $P$ value $<0.05$ was considered statistically significant.

\section{Results}

Out of 92 participants with enuresis, 30 children received nasal desmopressin, 31 children were given imipramine, and 31 others received oxybutynin. Three groups recruited for the study were rather homogenous. The characteristics of the groups are shown in Table 1.

During the 6-week-trial, pharmacotherapy was successful in 60 children, indicating the overall response rate of $65.2 \%$. The study revealed a slightly higher rate of response to treatment in the oxybutynin group (71.0\%), compared with the desmopressin group (63.3\%), and the imipramine group (61.3\%). However, the difference between the three groups was not statistically significant $(\mathrm{P}=0.70)$.

After discontinuing the therapy, 30 children presented with bed wetting again, indicating a relapse rate of $50 \%$ in general. The oxybutynin group showed a markedly lower relapse rate $(31.8 \%)$ compared to the other two groups (desmopressin 57.9\% and imipramine 63.2\%), however, the difference was not statistically significant $(P=0.095)$ (Table 2).

The present study found no significant side effects due to the medications. A few children in the oxybutynin group experienced mild headache, dry mouth, or stomach upset, which did not lead to discontinuation of the treatment. A small number of children in the imipramine group had mild drowsiness, and a few of them experienced mild gastrointestinal upset, which was not disturbing. Mild headache and minimal nasal congestion were the only side effects in the desmopressin group. As a whole, none of our samples suffered from noticeable side effects due to medications. 
Seyfhashemi Metal.



${ }^{\mathrm{a}}$ Data are presented as No. (\%) or mean \pm SD. 
Seyfhashemi Met al.

Table 2. Treatment Outcomes (Response and Relapse Rates) in Groups a

\begin{tabular}{lccccc}
\hline Variables & Whole sample $(\mathbf{n}=\mathbf{9 2})$ & Desmopressin $(\mathbf{n}=\mathbf{3 0})$ & Imipramine $(\mathbf{n}=\mathbf{3 1})$ & Oxybutynin $(\mathbf{n}=\mathbf{3 1})$ & PValue \\
\hline Response & $60(65.2)$ & $19(63.3)$ & $19(61.3)$ & $22(71.0)$ & 0.701 \\
Relapse & $30(50.0)$ & $11(57.9)$ & $12(63.2)$ & $7(31.8)$ & 0.095 \\
\hline
\end{tabular}

${ }^{\mathrm{a}}$ Data are presented as No. (\%).

\section{Discussion}

The study evaluated the treatment outcomes in children enuresis and compared the successful response and relapse rates among children treated with three different medications. Our aim was to assess which of the pharmacological interventions was more efficacious. We evaluated the outcomes of the interventions by assessing the rate of response, as well as the rate of relapse. No serious adverse effects were reported in any group.

\subsection{Rate of Response}

The study showed that oxybutynin was successful in $71 \%$ of children with enuresis after 6 weeks therapy, followed by desmopressin (63.3\% success) and imipramine in (61.3\% success). Although the response rate was higher in the oxybutynin group than the desmopressin and imipramine groups, the difference was not statistically significant $(P=0.701)$. A huge number of studies have compared the effect of non-pharmacological interventions, desmopressin, antidepressants, or various combination therapies. Review of the literature indicates that oxybutynin has been effective in $47-71 \%$ of children and showed a better response if combined with desmopres$\sin (2)$. Nasal desmopressin had been efficacious in 60 $70 \%$ and imipramine in $40-60 \%$ of children (2). A systematic review based on a meta-analysis from the Cochrane collaborative stated that desmopressin can result in 1 - 2 fewer wet nights per week compared with the placebo (8). Another systematic review showed that imipramine has been associated with a reduction of about one wet night per week during the treatment (9). There is not enough evidence-based and reliable information indicating oxybutynin as an efficacious treatment for monosymptomatic enuresis (10). Some studies recommended oxybutynin for desmopressin resistant children, or those with primary nocturnal enuresis and daytime wetting $(2,11-13)$. Several studies stated that oxybutynin is more effective in children who have small bladders, restricted bladder capacity, thickened bladder wall, and hyperactive detrusors $(11,14)$. In one study, the majority of children enuresis (88.2\%) with inadequate bladder storage function were responsive to the $15 \mathrm{mg}$ daily oxybutynin regime, while in patients with normal bladder function this medication was generally unsuccessful for enuresis (15). In a study by Montaldo et al. (11), the responders to combined oxybutynin and desmopressin showed a higher rate of response (45\%) compared with the desmopressin plus placebo group (17\%).
In a recent study in Iran, the cure rate for oxybutynin was only $23 \%$ (16), while in a randomized double-blind controlled trial the response rate of oxybutynin was $45 \%$ (11). Our study showed that oxybutynin has been more effective than the other pharmacological options. Oxybutynin had a role for treating children with a restricted bladder capacity who did not have lower urinary tract symptoms during the day (17). One study concluded that in children with enuresis, bladder capacity during sleep was significantly lower than day time capacity, and these children may not be able to hold urine during sleep (18). This may explain the better response to oxybutynin in our samples. We did not evaluate children's bladder capacity or function, but we obtained a better response to oxybutynin, which was inconsistent with previous studies. Yeung et al. observed a reduction in nocturnal functional capacity of bladder during sleep at night in children who had a normal daytime urodynamic and functional bladder capacity. These children were refractory to treatment with desmopressin (19), but they showed a good response to oxybutynin $(12,15,17)$. Neveus $(14)$ in a case-control study concluded that those responding to oxybutynin have small bladders and possibly hyperactive detrusors, whereas children responding to desmopressin or those who need both drugs for staying dry have polyuria. Our findings highlight the idea that children with monosymptomatic enuresis without lower urinary tract symptoms during the day may still have problems with their nocturnal or functional bladder capacity.

It is well established that treating enuresis cannot be successful if the child and their parents do not cooperate, and that treatment with medication will fail if the family's social construct and home circumstances are not supportive. The clear differences between the findings of our study and previous studies may be partly explained by this point. Other important factors in treatment success are comorbid psychological, behavioral, and emotional problems as well as age of the child (9). A positive family history of enuresis, child's drinking habit, abnormal deep sleep pattern and constipation have a strong impact on the response to treatment (20). Parents, who experienced bedwetting, may have a more tolerant attitude towards the enuresis of their children (20), which can positively affect the child's response to the medication. Other risk factors of enuresis, including low socioeconomic level of the family, low school success, inappropriate and forced toilet training, and strict or over protective parents (21) 
Seyfhashemi Met al.

may also influence the response to treatment. Moreover, the various treatment approaches are influenced by the child's environment, and by the background and prejudgments of the family. As suggested by the Cochrane Database of systematic reviews: "not all interventions are suitable for all children" (9).

\subsection{Rate of Relapse}

In our study, the oxybutynin group showed a clearly lower relapse rate (31.8\%) compared to the desmopressin (57.9\%) and imipramine (63.2\%) groups, with the relapse rate in the imipramine group being approximately twice as much as of the oxybutynin group. However, the difference was not statistically significant $(P=0.095)$. There are limited studies on relapse rates of anticholinergic medication in enuresis. However, studies evaluating other medications have shown good initial response, albeit a substantial relapse rate (80 - 90\%) when children were off medication (1). Studies on desmopressin have reported the relapse rates as high as $80 \%$ (22), which is higher than the relapse rate in our study. Some RCTs have shown no significant difference between desmopressin and placebo in terms of success rates after discontinuing the treatment; and in comparison with alarms, desmopressin has been less effective when treatment was stopped (7). Review of the literature has shown that most children would relapse after discontinuing imipramine treatment (9). Controlled double blind trials have reported a long-term cure rate of $25 \%$ for imipramine (23). Relapse was reported in $60 \%$ of patients with enuresis treated with oxybutynin plus imipramine during the follow up period (12). Only in one study, the relapse rates for imipramine and oxybutynin were $58.6 \%$ and $42.3 \%$, respectively (16), which was compatible to our findings. In addition, the study conducted by Kwak KW and his co-workers (24) showed that in the full responders to desmopressin and enuresis alarm, 60.7\% did not relapse after cessation of treatment. However, when they added oxybutynin, 88.9\% did not relapse after discontinuation of treatment. These findings are also in agreement with the results of our study.

The main limitation of our study was the small sample size. Although the relapse rate of the three groups were different, because of the small sample size, the statistical analysis was not significant. The other limitation was that we recruited our samples from a relatively homogenous urban population with rather similar cultural and family circumstances. Further clinical trial studies with larger sample size on different populations are needed to produce more reliable results in this field.

Nocturnal enuresis is a common problem seen by pediatricians and is one of the most common sources of concern for children and their families. Despite many years of research, there are still some uncertainties about the benefit of pharmacological treatment and the most efficacious interventions. More studies with larger sample size and longer duration of treatment and follow up are needed to decide which treatment is the most appropriate for enuresis. Studies should be performed on children with various cultural and socioeconomic backgrounds and different family circumstances and should take into account the individual characteristic of every child. This will help clinicians to determine which treatment best suites which children.

\section{Authors' Contributions}

Study concept and design: Maryam Seyfhashemi; Acquisition of data and drafting of the manuscript, as well as administrative, technical, and material support: Abbas Zolfaghari and Maryam Seyfhashemi; Analysis and interpretation of data: Raheb Ghorbani and Maryam Seyfhashemi; Statistical analysis: Raheb Ghorbani; Study supervision: Maryam Seyfhashemi; and Critical revision of the manuscript for important intellectual content: All authors.

\section{Financial Disclosure}

None of the authors had any financial interests related to the material in the manuscript, or any conflict of interest to declare.

\section{Funding/Support}

This work was financially supported by Semnan University of Medical Sciences, Semnan, IR Iran.

\section{References}

1. Lawless MR, McElderry DH. Nocturnal enuresis: current concepts. Pediatr Rev. 2001;22(12):399-407.

2. Ramakrishnan K. Evaluation and treatment of enuresis. Am Fam Physician. 2008;78(4):489-96.

3. American Psychiatric Association. Diagnostic and statistical manual of mental disorders: DSM-IV [Internet]. Washington: 1994.

4. Kiddoo D. Nocturnal enuresis. BMJ Clin Evid. 2011;2011.

5. Forsythe WI, Redmond A. Enuresis and spontaneous cure rate. Study of 1129 enuretis. Arch Dis Child. 1974;49(4):259-63.

6. Feldman M. Management of primary nocturnal enuresis. Paediatr Child Health. 2005;10(10):611-4.

7. Makari J, Rushton HG. Nocturnal enuresis. Am Fam Physician. 2006;73(9):1611-3.

8. Glazener CMA, Evans JHC. Desmopressin for nocturnal enuresis in children. Cochrane Database Syst Rev. 2002;3(CD002112).

9. Glazener CMA, Evans JHC, Peto RE, Glazener CMA. Tricyclic and related drugs for nocturnal enuresis in children. Cochrane Database Syst Rev. 2003;3:CD002117.

10. Deshpande AV, Caldwell PH, Sureshkumar P. Drugs for nocturnal enuresis in children (other than desmopressin and tricyclics). Cochrane Database Syst Rev. 2012;12:CD002238.

11. Montaldo P, Tafuro L, Rea M, Narciso V, Iossa AC, Del Gado R. Desmopressin and oxybutynin in monosymptomatic nocturnal enuresis: a randomized, double-blind, placebo-controlled trial and an assessment of predictive factors. BJU Int. 2012;110(8 Pt B):381-6.

12. Friedman BC, Friedman B, Goldman RD. Oxybutynin for treatment of nocturnal enuresis in children. Can Fam Physician. 2011;57(5):559-61.

13. Radvanska E, Kovacs L, Rittig S. The role of bladder capacity in antidiuretic and anticholinergic treatment for nocturnal enuresis. JUrol. 2006;176(2):764-8.

14. Neveus T. Oxybutynin, distance and enuresis. JUrol. 2001;166(6):2459-62. 


\section{Seyfhashemi Met al.}

15. Kosar A, Arikan N, Dincel C. Effectiveness of oxybutynin hydrochloride in the treatment of enuresis nocturna--a clinical and urodynamic study. Scand J Urol Nephrol. 1999;33(2):115-8.

16. Esmaeili M. Combined treatment with oxybutynin and imipramine in enuresis. Iran J Med Sci. 2015;33(1):12-6.

17. Austin PF, Ferguson G, Yan Y, Campigotto MJ, Royer ME, Coplen DE. Combination therapy with desmopressin and an anticholinergic medication for nonresponders to desmopressin for monosymptomatic nocturnal enuresis: a randomized, double-blind, placebo-controlled trial. Pediatrics. 2008;122(5):1027-32.

18. Kawauchi A, Tanaka Y, Naito Y, Yamao Y, Ukimura O, Yoneda K, et al Bladder capacity at the time of enuresis. Urology. 2003;61(5):1016-8.

19. Yeung CK, Sit FK, To LK, Chiu HN, Sihoe JD, Lee E, et al. Reduction in nocturnal functional bladder capacity is a common factor in the pathogenesis of refractory nocturnal enuresis. BJU Int. 2002;90(3):302-7.
20. Neveus T, Eggert P, Evans J, Macedo A, Rittig S, Tekgul S, et al. Evaluation of and treatment for monosymptomatic enuresis: a standardization document from the International Children's Continence Society. JUrol. 2010;183(2):441-7.

21. Ozkan S, Durukan E, Iseri E, Gurocak S, Maral I, Ali Bumin M. Prevalence and risk factors of monosymptomatic nocturnal enuresis in Turkish children. Indian J Urol. 2010;26(2):200-5.

22. Fritz G, Rockney R, Bernet W, Arnold V, Beitchman J, Benson RS, et al. Practice parameter for the assessment and treatment of children and adolescents with enuresis. J Am Acad Child Adolesc Psychiatry. 2004;43(12):1540-50.

23. Cendron M. Primary nocturnal enuresis: current. Am Fam Physician.1999;59(5):1205-14.

24. Kwak KW, Park KH, Baek M. The efficacy of enuresis alarm treatment in pharmacotherapy-resistant nocturnal enuresis. Urology. 2011;77(1):200-4. 